\title{
Reabilitação funcional e estética de dente anterior fraturado em paciente hebiátrico: relato de caso
}

Functional and aesthetic rehabilitation of fractured anterior teeth in a hebiatric patient: case report Rehabilitación funcional y estética de diente anterior fracturado en paciente hebiátrico: relato de caso

Carlos Rodrigo Jordão de ALBUQUERQUE ${ }^{\mathbf{1}}$ Diego Filipe Bezerra SILVA ${ }^{2}$ Olímpia Crispim da SILVEIRA ${ }^{3}$

Carmen Lúcia Soares Gomes de MEDEIROS ${ }^{3}$ Maria Helena Chaves de Vasconcelos CATÃO ${ }^{4}$

${ }^{1}$ Graduado em Odontologia pela Universidade Estadual da Paraíba-UEPB, 58429-600, Campina Grande-PB, Brasil ${ }^{2}$ Mestrando do Programa de Pós-Graduação em Odontologia da Universidade Estadual da Paraíba-UEPB, 58429-600, Campina Grande-PB, Brasil ${ }^{3}$ Professora Doutora do curso de Odontologia da Universidade Estadual da Paraíba - UEPB, 58429-600, Campina Grande-PB, Brasil

${ }^{4}$ Professora Doutora do Programa de Pós-Graduação em Odontologia da Universidade Estadual da Paraíba-UEPB, 58429-600, Campina Grande-PB, Brasil

\section{Resumo}

Introdução: Após fratura decorrente de trauma e consequente tratamento endodôntico, os dentes têm sua resistência mecânica reduzida, sendo necessária, em alguns casos, a utilização de um retentor intrarradicular. Objetivo: Descrever um caso clínico de reabilitação de um dente anterior que apresentava fratura decorrente de trauma, de um paciente adolescente. Relato do caso: Paciente do sexo masculino, 15 anos de idade, apresentou-se com a queixa da aparência estética do dente 32 . Na anamnese o paciente relatou que há cerca de 1 ano havia fraturado o referido dente enquanto brincava, e que sentiu dor apenas nas primeiras horas após o acidente, não se queixando mais de qualquer sintomatologia dolorosa desde então. Ao exame radiográfico, observou-se fratura do dente 32, além da presença de imagem radiolúcida na região periapical do mesmo dente. Assim, baseado na avaliação radiográfica e após criteriosos exames com recursos semiotécnicos empregados na Endodontia, confirmou-se o diagnóstico de necrose pulpar no referido dente. Foi instituído o tratamento endodôntico previamente ao tratamento restaurador. A reabilitação consistiu inicialmente pelo tratamento endodôntico. Na sequência optou-se pela instalação de um pino de fibra de vidro (PFV). Por fim, a reconstrução coronária foi realizada de forma direta, em resina composta e com o auxílio de uma coroa de cloreto de polivinila (PVC). Conclusão: Através do resultado final obtido, concluiu-se que esta é uma alternativa rápida e viável para casos como o apresentado, proporcionando não somente o restabelecimento funcional e estético do dente, mas também satisfação e elevação da autoestima de pacientes hebiátricos.

Descritores: Traumatismos Dentários; Dente não Vital; Restauração Dentária Permanente.

\section{Abstract}

Introduction: After fracture resulting from trauma and consequent endodontic treatment, the teeth have reduced mechanical resistance, and in some cases, the use of an intraradicular retainer is necessary. Objective: To describe a case report of rehabilitation of anterior tooth who presented a fracture due to trauma of a hebiatric patient. Case report: A 15-year-old male presented with the complaint of the aesthetic appearance of the tooth 32. During the anamnesis, the patient reported that about 1 year had fractured said tooth while playing, and who felt pain only in the first few hours after the accident, not complaining any more of any painful symptomatology ever since. Radiographic examination revealed a fracture of the tooth 32 , besides the presence of a radiolucent image in the periapical region of the same tooth. Thus, based on the radiographic evaluation and after careful examinations with semiotechnical resources used in Endodontics, the diagnosis of pulp necrosis in this tooth was confirmed. Endodontic treatment was instituted prior to restorative treatment. The rehabilitation initially consisted of endodontic treatment. In the sequence, a fiberglass pin (FGP) was installed. Finally, the direct coronary reconstruction was performed, in a composite resin and with the aid of a crown of polyvinyl chloride (CPC). Conclusion: Through the final result obtained, it was concluded that this is a fast and viable alternative for cases such as the one presented, providing not only the functional and aesthetic restoration of the dental element, but also satisfaction and elevation of self-esteem of hebiatric patients.

Descriptors: Tooth, Injuries; Tooth, Nonvital; Dental Restoration, Permanent.

\section{Resumen}

Introducción: Después de la fractura resultante de trauma y consecuente tratamiento endodóntico, los dientes tienen su resistencia mecánica reducida, siendo necesaria, en algunos casos, la utilización de un retén intrarradicular. Objetivo: Describir un caso clínico de rehabilitación de un diente anterior que presentaba fractura resultante de trauma, de un paciente adolescente. Relato del caso: Paciente del sexo masculino, 15 años de edad, se presentó con la queja de la apariencia estética del diente 32. En el anamnesis, el paciente relató que hace cerca de 1 año, había fracturado el referido diente mientras jugaba, y, en el caso de la mujer, que sintió dolor sólo en las primeras horas después del accidente, no se quejó más de cualquier sintomatología dolorosa desde entonces. En el examen radiográfico, se observó fractura del diente 32, además de la presencia de imagen radiolúcida en la región periapical del mismo diente. Así, basado en la evaluación radiográfica y después de criteriosos exámenes con recursos semiotécnicos empleados en la Endodoncia, se confirmó el diagnóstico de necrosis pulpar en el referido diente. Se ha instituido el tratamiento endodóntico previamente al tratamiento restaurador. La rehabilitación consistió inicialmente en el tratamiento endodóntico. En la secuencia se optó por la instalación de un pasador de fibra de vidrio (PFV). Por último, la reconstrucción coronaria fue realizada de forma directa, en resina compuesta y con el auxilio de una corona de cloruro de polivinilo (PVC). Conclusión: A través del resultado final obtenido, se concluyó que esta es una alternativa rápida y viable para casos como el presentado, proporcionando no sólo el restablecimiento funcional y estético del diente, sino también satisfacción y elevación de la autoestima de pacientes hebiátricos.

Descriptores: Traumatismos de los Dientes; Diente no Vital; Restauración Dental Permanente.

\section{INTRODUÇÃO}

A prevalência de fraturas coronárias em dentes anteriores é considerada alta, tendo uma incidência de $1 \%$ a $3 \%$ na população mundial ${ }^{1}$. Cerca de $75 \%$ dos casos são em dentes permanentes e um dos seus principais responsáveis é o traumatismo dentário $(\mathrm{TD})^{2}$. Este acomete principalmente indivíduos na fase da hebiatria, que abrange dos 10 aos 20 anos de idade ${ }^{3}$. Traumatismos em dentes anteriores na dentição decídua ou permanente requerem um tratamento funcional rápido e estético, pois podem provocar danos irreparáveis, afetando as condições psicológicas, sociais e estéticas do indivíduo. Portanto, essas situações comumente se tornam experiências dramáticas para o paciente, e o cirurgião-dentista deve estar apto a realizar os procedimentos necessários ${ }^{4,5}$. 
Dependendo da intensidade da injúria provocada por um TD, repercussões sobre o tecido pulpar são frequentes. Após 48 horas do trauma, a possibilidade de contaminação bacteriana da polpa aumenta na medida em que a inflamação progride para o ápice ${ }^{6}$. Assim, com o passar do tempo a chance de manutenção de uma polpa saudável diminui, e a necrose pulpar é uma sequela comumente encontrada, se desenvolvendo dentro do primeiro ano após o trauma ${ }^{7}$. Nesse caso, é necessário realizar o tratamento endodôntico do dente previamente à sua restauração.

Quando há grande perda de estrutura dentinária, a restauração deve ser planejada para proteger o remanescente contra fraturas e substituir a estrutura dental perdida. Desta forma, muitas vezes é necessário instalar no dente um dispositivo intrarradicular capaz de aumentar a retenção do conjunto formado pelo tecido dental remanescente e o material restaurador. Os pinos intrarradiculares são dispositivos pré-fabricados que são cimentados em dentes tratados endodonticamente com a finalidade de aumentar a retenção das restaurações. Eles são indicados justamente para os casos em que o tecido dentário remanescente é insuficiente ou não tem condições de promover adequado suporte e/ou retenção para a restauraçãa ${ }^{8}$.

Os pinos de fibra de vidro (PFV) préfabricados foram introduzidos no mercado para substituir os pinos metálicos, tendo se popularizado cada vez mais. Sua utilização associada à restaurações diretas tem sido frequente e alcançado prognósticos favoráveis. Eles são compostos por uma matriz resistente de resina composta, na qual são adicionadas fibras longitudinais de vidro a fim de melhorar suas propriedades mecânicas 9 .

Apesar de simples, a técnica de utilização do retentor de fibra de vidro deve ser realizada criteriosamente, sem negligência em nenhuma das suas etapas clínicas. Primeiro é necessário selecionar o diâmetro, o comprimento e a forma do pino que será utilizado. A desobstrução do canal radicular deve ser apenas parcial, preservando-se o devido selamento apical. Tanto o pino quanto o conduto devem passar por um tratamento de superfície ${ }^{9}$. Após a cimentação do pino no conduto, dá-se início à restauração coronária, de tal forma que o pino fique totalmente protegido do meio bucal ${ }^{10}$.

Dessa forma, o objetivo do presente trabalho é relatar um caso clínico em que a reabilitação de um dente anterior de um paciente adolescente foi realizada por meio de restauração direta em resina composta associada à instalação de um retentor intrarradicular de fibra de vidro.

\section{CASO CLÍNICO}

Paciente do sexo masculino, 15 anos de idade, leucoderma, acompanhado da responsável,

procurou a Clínica Escola de Odontologia da Universidade Estadual da Paraíba (UEPB, Campina Grande/PB) queixando-se da aparência estética de um dos seus dentes. $\mathrm{Na}$ anamnese, o paciente relatou que há cerca de 1 ano, havia fraturado um dente quando brincava, sentindo dor apenas nas primeiras horas após o acidente, não se queixando de qualquer sintomatologia dolorosa desde então.

Durante o exame clínico foi constatada uma fratura envolvendo esmalte e dentina no dente $32 \mathrm{e}$ leve extrusão e deslocamento palatino do dente 21 . $\mathrm{Na}$ radiografia periapical inicial, ficou evidenciada a fratura do dente 32 (Figura 1A), além da presença de imagem radiolúcida na região periapical do mesmo dente (Figura 1B). Assim, baseado na avaliação radiográfica e após criteriosos exames com recursos semiotécnicos empregados na Endodontia, confirmou-se o diagnóstico de necrose pulpar no referido dente. Foi instituído o tratamento endodôntico previamente ao tratamento restaurador.

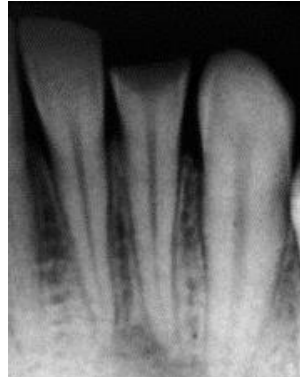

A

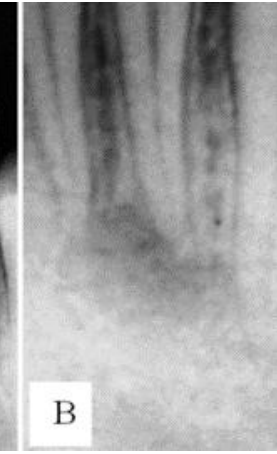

Figura 1: (A) Imagem radiográfica evidenciando fratura coronária envolvendo esmalte e dentina do dente 32. (B) Imagem radiolúcida no periápice do dente 32 .

Para o preparo do canal radicular foi empregada a técnica de Oregon (Crown-Down) modificada. $\mathrm{Na}$ primeira sessão, realizou-se a abertura coronária, preparo dos terços cervical e médio, odontometria, preparo químico-mecânico (Figura 2A) e inserção de medicação intracanal à base de hidróxido de cálcio. Na segunda e última sessão do tratamento endodôntico, obturou-se o canal radicular empregando-se a técnica de condensação lateral (Figura 2B). Por fim, realizou-se o selamento coronário com cimento de ionômero de vidro (Figura 2C).

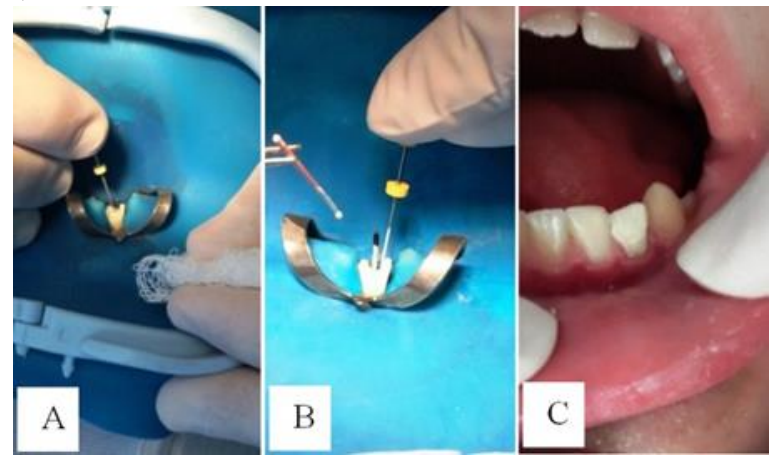

Figura 2. Tratamento endodôntico do dente 32. (A) Preparo químico-mecânico. (B) Obturação do canal radicular. (C) Selamento coronário provisório. 
Após a conclusão do tratamento endodôntico, foi avaliada radiograficamente a espessura presente de dentina radicular e, considerando esse fator, bem como o comprimento da raiz e sua inclinação, a anatomia do conduto e a estrutura coronária remanescente (Figura 3), optou-se por, numa nova sessão, realizar a técnica de restauração direta em resina composta associada à instalação de um PFV.

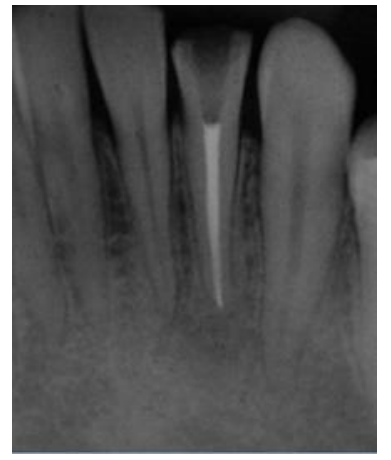

Figura 3. Aspecto radiográfico do canal radicular do dente 32 obturado.

$\mathrm{Na}$ sessão seguinte, realizou-se profilaxia com pedra pomes/água e escova Robinson, seguida de taça de borracha. A seleção da resina composta foi realizada pela técnica da visualização direta, utilizando a escala VITA, sendo a cor A 3,5 (FGM, Joinville, SC, Brasil) a escolhida para a restauração do dente. Em seguida, removeu-se o selamento provisório para acessar e avaliar clinicamente o diâmetro e o formato do canal radicular (Figura 4). Após isolamento do campo operatório, desobturou-se $10 \mathrm{~mm}$ de um total de $14 \mathrm{~mm}$ obturados do conduto, utilizando broca Largo Peeso (Figuras 5A e 5B). Um condensador levemente aquecido foi utilizado para auxiliar na manutenção de $4 \mathrm{~mm}$ do material obturador na porção apical e na remoção de resíduos das paredes do conduto, que poderiam comprometer a etapa de cimentação do pino.

Foi selecionado pino cônico $\mathrm{n}^{\circ} 1$ de dupla conicidade (Whitepost DC, FGM, Joinville-SC, Brasil). Após testar clinica e radiograficamente sua adaptação à extensão desobturada do conduto (Figuras 6A e 6B), o pino teve seu comprimento reduzido de tal forma que, quando adaptado ao canal, sua extremidade coronária ficasse $2 \mathrm{~mm}$ aquém da borda incisal do dente. Para tal, utilizou-se ponta diamantada tronco-cônica (2135, KG Sorensen, Cotia, SP, Brasil) refrigerada com jato spray ar/água em alta rotação (Figura 6C). Em seguida o pino foi limpo com gel de ácido fosfórico $37 \%$ durante 30 segundos, removendo detritos e oleosidade da sua superfície. O tratamento de superfície foi realizado com a aplicação do agente de união silano (Maquira, Maringá-PR, Brasil) em toda sua extensão (Figura 7A), aguardando 60 segundos, seguida de secagem com jato de ar, aplicação de camada do sistema adesivo (Maquira, Maringá-PR, Brasil) (Figura 7B) e fotoativação.

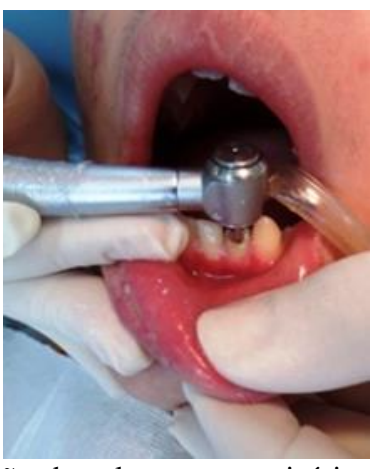

Figura 4. Remoção do selamento provisório e acesso ao canal radicular.

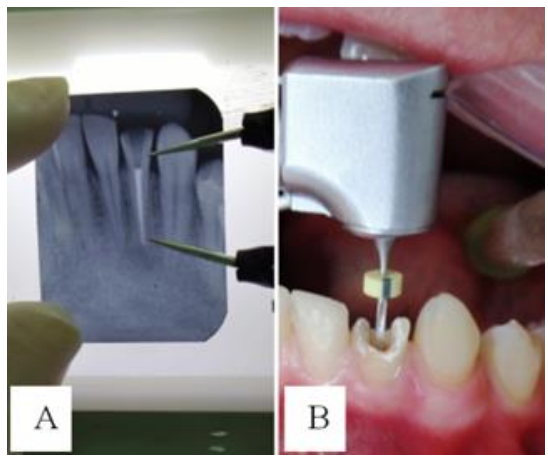

Figura 5. (A) Definição da extensão do conduto a ser desobturada. (B) Desobturação parcial do canal.

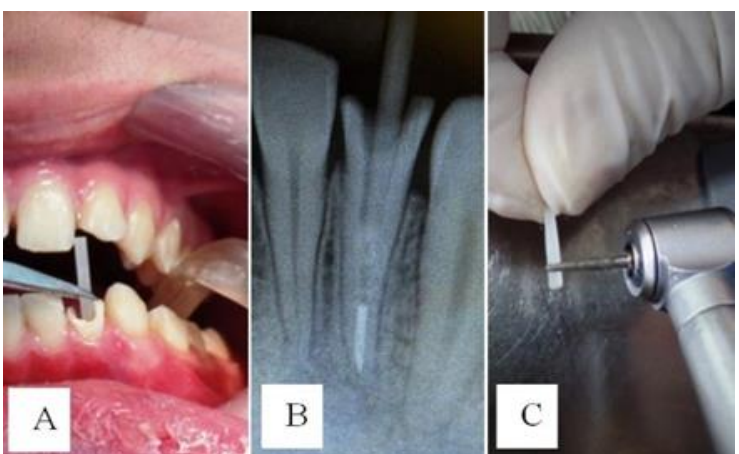

Figura 6. (A) Teste de adaptação do PFV ao canal radicular. (B) Corte axial do pino a partir da sua extremidade coronária para redução do comprimento.
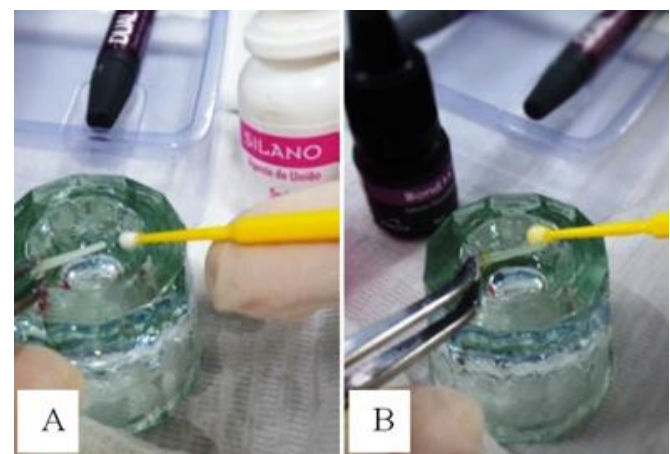

Figura 7. (A) Tratamento de superfície do PFV com o agente de união Silano. (B) Aplicação de sistema adesivo no PFV.

$\mathrm{Na}$ sequência, as paredes do conduto receberam tratamento com gel de ácido fosfórico $37 \%$ durante 15 segundos (Figura 8A), seguido de lavagem com jato d'água pelo mesmo intervalo de tempo e secagem com cone de papel absorvente. Aplicou-se o sistema adesivo (Maquira, Maringá, PR, Brasil) (Figura 8B), fotoativando em seguida por 30 segundos (Figura 8C). 


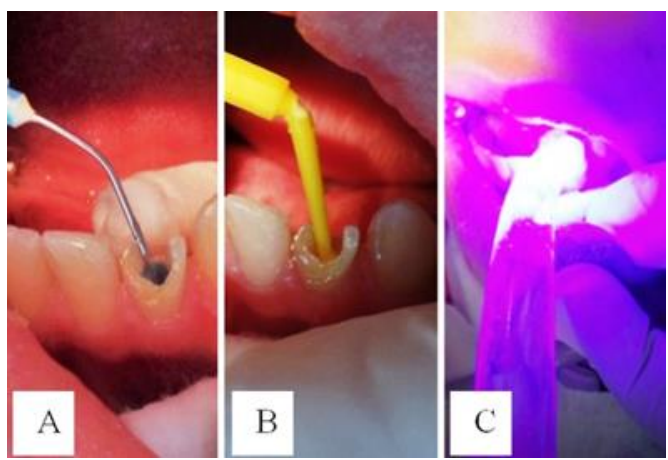

Figura 8. (A) Tratamento com ácido fosfórico 37\% nas paredes do conduto radicular. (B) Aplicação do sistema adesivo. (C) Fotopolimerização.

Para a cimentação do pino, utilizou-se o cimento resinoso (Fill Magic Dual, Coltene, Rio de Janeiro, RJ, Brasil), manipulando conforme as orientações do fabricante (figura 9A). Com o auxílio do próprio pino e simultaneamente com ele, o cimento foi inserido no conduto (Figura 9B), removendo-se o excesso com espátula, e fotopolimerizado por 60 segundos (Figura 9C). Finalizando esta etapa, uma nova radiografia foi feita para constatação da adaptação do pino, agora cimentado ao conduto.

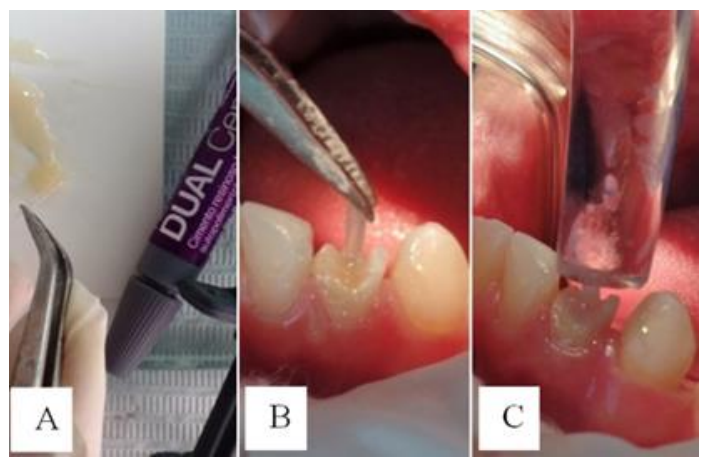

Figura 9. (A) Manipulação do cimento resinoso. (B) Inserção simultânea do PFV e do cimento no conduto radicular. (C) Fotopolimerização do cimento.

$\mathrm{Na}$ mesma sessão clínica, foi realizada a restauração direta com a resina composta previamente selecionada, empregando-se de início a técnica incremental para encobrir por completo toda a porção coronária do pino (Figura 10A). Após isso, o procedimento restaurador foi sequenciado com o auxílio de uma coroa transparente de cloreto de polivinila (PVC) (TDV, Pomerode, SC, Brasil) (Figura 10B), a qual recebeu ajustes para se adaptar à anatomia do dente. Após testar essa adaptação (Figura 10C), a coroa de PVC foi preenchida com resina (Figura 11A) e inserida no dente até alcançar a reconstrução anatômica do mesmo (Figura 11B), removendo-se o excesso da resina extravasado por um furo previamente feito na sua face lingual. Após fotoativação por 40 segundos, a coroa de PVC foi removida com auxílio de uma sonda exploradora (Figura 11C).

Durante o ajuste oclusal, houve a necessidade de deixar a restauração com altura levemente reduzida devido ao toque prematuro no ângulo distoincisal do elemento 21. Com auxílio de um carbono de articulação, realizou-se o registro oclusal (Figura $12 \mathrm{~A}$ e 12B) e posterior desgaste e ajuste da restauração, utilizando pontas diamantadas de granulação extrafina (Figura 12C).

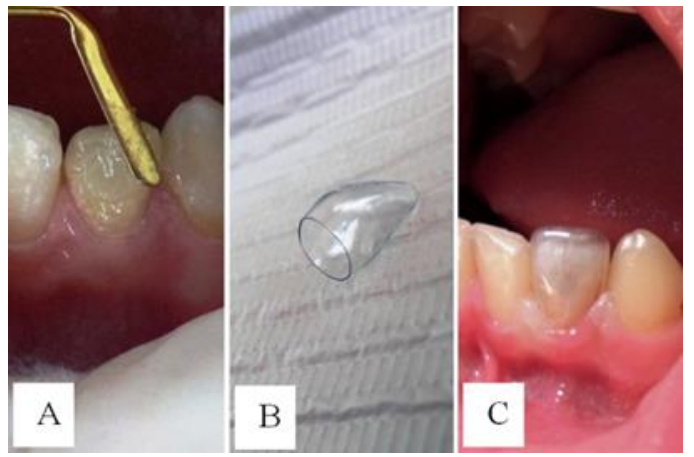

Figura 10. (A) Incrementos de resina composta; (B) Coroa transparente anterior de PVC). (C) Teste de adaptação da coroa de PVC.

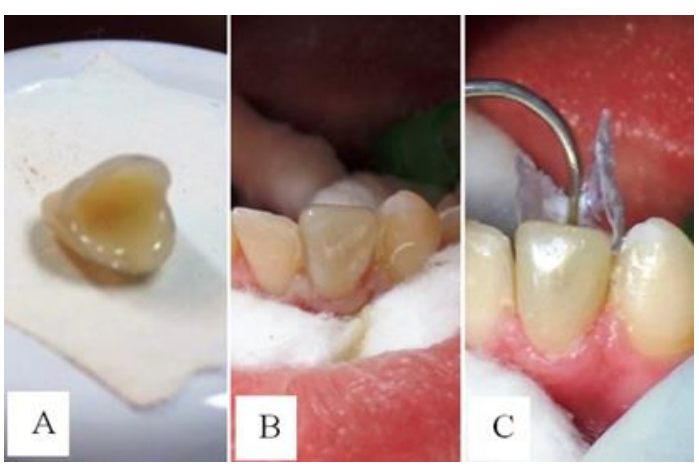

Figura 11. (A) Coroa transparente de PVC preenchida com a resina composta, e (B) inserida no dente. (C) Remoção da coroa de PVC após fotoativação.
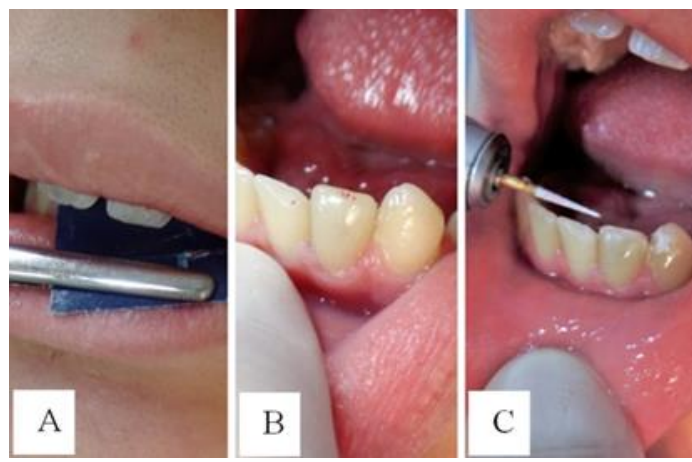

Figura 12. (A) e (B) Registro oclusal. (C) Desgaste e ajuste da restauração.

Uma última tomada radiográfica também foi realizada para avaliação final (Figura 13A). Após 72 horas, realizou-se um leve polimento na face incisal do dente, utilizando borrachas abrasivas/pasta polidora em baixa rotação. Ao final do tratamento, o paciente demonstrou satisfação no que tange ao resultado obtido (Figuras 13B e 13C). Ainda assim, foi recomendado tratamento ortodôntico para que, uma vez corrigida a oclusão, seja possibilitado o restabelecimento da altura cérvico-incisal ideal do elemento tratado (já que o mesmo foi restaurado em sua altura aquém do elemento adjacente devido ao leve toque prematuro no dente 21). Por fim, foram 
passadas instruções de higiene oral ao paciente e a sua responsável, bem como marcada uma reavaliação para controle após dois meses.

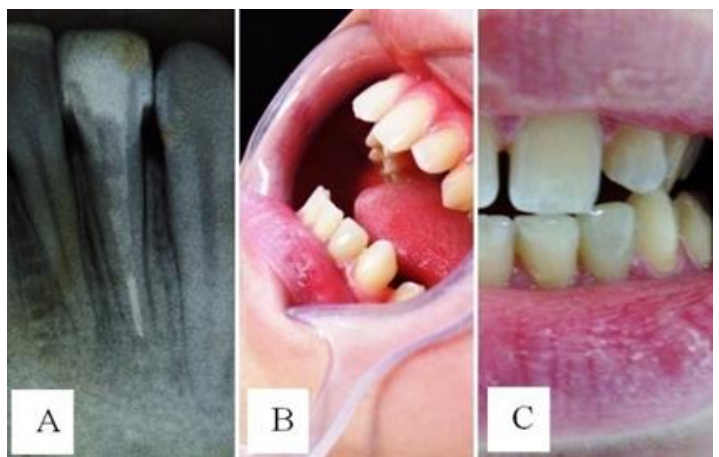

Figura 13. (A) Aspecto radiográfico final. (B) e (C) Resultado clínico final do restabelecimento estético-funcional do dente 32 .

DISCUSSÃO

Após um tratamento endodôntico, a restauração de um dente anterior é uma etapa essencial para o sucesso clínico do tratamento, pois além de restabelecer a função ao dente, também se espera que o remanescente dental fique protegido de fraturas e uma possível recontaminação do canal radicular seja evitada. A reconstrução, nesse caso, deve ter como propósito não somente substituir a estrutura dental perdida por um material restaurador, mas também fornecer uma resistência adequada ao remanescente fragilizado.

No caso clínico ora apresentado, havia a necessidade de se utilizar um meio de retenção intrarradicular que promovesse estabilidade ao procedimento restaurador, tendo em vista o comprometimento de estrutura coronária e, principalmente, o desgaste de dentina após o preparo químico mecânico do canal. O material restaurador precisava de um meio que o mantivesse retido ao remanescente dentário enfraquecido, proporcionando-lhe maior estabilidade e apoio interno.

Deve-se considerar também que o dente em questão é anterior (incisivo lateral inferior), cuja incidência de forças transversais é uma constante durante suas funções mastigatórias e até mesmo hábitos parafuncionais, o que pode comprometer a estrutura de suporte fragilizada. Nesse caso, torna-se imprescindível o conhecimento de princípios mecânicos e das propriedades do material escolhido para a restauração do referido dente $\mathrm{e}^{11,12}$.

Deve-se ressaltar que a seleção do meio de retenção mais adequado para o caso pode interferir diretamente na longevidade do tratamento. Para a obtenção de um prognóstico favorável, a literatura aponta que a porção coronária do dente deve ter pelo menos 2,0 mm de tecido dental. Aliado a este prérequisito, a condição do remanescente dentário e a estética (principalmente por se tratar de um dente anterior) também foram fatores decisivos para a escolha do PFV no caso relatado.
Além de biocompatível e proporcionar a desejada retenção do material restaurador, o PFV tem aplicação clínica que demanda tempo reduzido quando comparado ao uso de outros sistemas. Ele já é fabricado num formato compatível com a morfologia do conduto do elemento aqui tratado, o que deve proporcionar alta retentividade mecânica e menor desgaste dentinário na porção apical ${ }^{13}$. Também se pode destacar como características dos PFV: alta resistência à corrosão e à fratura; compatibilidade com sistemas adesivos e cimentos resinosos, apresentando excelente adesão dente/cimento/material de preenchimento; translucidez; alta radiopacidade; baixa absorção de água e solubilidade ${ }^{14}$.

A característica mais destacada do PFV é o seu comportamento mecânico, já que diferente de um núcleo metálico fundido, por exemplo, ele amortece vibrações e absorve choques, poupando a raiz de uma sobrecarga maior durante o esforço oclusal, o que diminui a incidência de fraturas radiculares. Esse diferencial é explicado pelo seu módulo de elasticidade semelhante ao da dentina, resultando numa melhor distribuição de cargas mastigatórias ao longo das paredes radiculares, quando comparado ao emprego da maioria dos outros tipos de retentores ${ }^{15}$. Feuser $^{14}$ atribui essa redução de fraturas ao comportamento dito anisotrópico dos PFV, alegando que seu módulo de elasticidade, além de baixo, varia em função da direção das cargas.

Como vantagens, o PFV dispensa a etapa laboratorial, o que reduz custos; demanda pouco desgaste dental para sua instalação, e proporciona uma estética favorável, já que transmite luz e possui coloração semelhante à da estrutura dental ${ }^{9}$. Outro fator relevante é que, em caso de uma reintervenção endodôntica, o PFV pode ser facilmente removido do canal radicular ${ }^{16}$.

As possíveis falhas desse sistema têm incidência reduzida e normalmente são relacionadas à fratura coesiva da resina composta ou descolamento do conjunto pino/reconstrução. De acordo com Sorrentino et al. ${ }^{17}$, essas falhas são reversíveis. Corroborando, alguns estudos mostraram que dentes reabilitados com PFV que sofreram alguma fratura, em sua maioria a estrutura dental remanescente foi preservada, possibilitando uma nova restauração. Já em dentes reabilitados com núcleos metálicos fundidos, por exemplo, há uma frequência maior de perdas do dente ${ }^{18}$.

Considerando que o risco de infiltração coronária é maior antes da restauração definitiva, esta foi realizada já na sessão seguinte à conclusão do tratamento endodôntico para que obtivéssemos mais previsibilidade de sucesso, conforme recomendado por Schwartz e Fransman ${ }^{19}$. Para uma maior possibilidade de reparação diante de possíveis falhas, deve-se destacar também a importância de um 
comprimento adequado do pino. Assim, o comprimento do preparo intrarradicular deve envolver $2 / 3$ ou $3 / 4$ do comprimento radicular, mantendo-se no terço apical de 3 a 4 milímetros de material obturador para garantir o devido selamento naquela região ${ }^{11}$.

Quanto ao seu diâmetro, vale ressaltar que o mesmo interfere na resistência à torção e, consequentemente, na sua retenção ao conduto. Quanto maior o diâmetro, mais íntimo será o contato do cimento com as paredes do canal e menor a chance de deslocamento do pino. Essa condição de boa adaptação do pino ao conduto também implica numa menor espessura da película de cimento, evitando a formação de bolhas que poderiam provocar falhas na cimentação e, consequentemente, diminuição da resistência à fratura ${ }^{11,20}$. Por esse motivo os PFV são contra-indicados em canais muito amplos e com grande desgaste ${ }^{21}$. Para estes casos, uma das técnicas propostas é o emprego de pinos anatômicos, através do reembasamento e moldagem do conduto radicular com resina composta associada ao pino, melhorando assim a adaptação e, consequentemente, possibilitando uma camada mais fina e uniforme de cimento ${ }^{22}$.

Apesar de se tratar de um incisivo inferior, cujas raízes são frequentemente achatadas no sentido mésio-distal, no caso ora apresentado o conduto apresentou-se relativamente cônico. Com isso, buscando uma adaptação satisfatória, o pino escolhido foi o de formato cônico com dupla conicidade. Conceição ${ }^{23}$ afirma que esse formato, assim como o cônico, exige menor desgaste de dentina radicular.

O preparo do pino antes da cimentação auxilia na limpeza da sua superfície e propicia um aumento no seu poder retentivo. $\mathrm{O}$ agente silano aumenta a molhabilidade superficial do pino, resultando na formação de ligações químicas com o grupo hidroxila da superfície das fibras de vidro. Além disso, ele promove união química entre as fibras de vidro expostas e o cimento resinoso ${ }^{3}$.

Para a cimentação do PFV no canal radicular são utilizados materiais restauradores adesivos que proporcionam união às paredes do conduto através de sistemas adesivos e cimentos resinosos. A cimentação adesiva com cimento resinoso deve ser a técnica de eleição para estes tipos de pino por formar uma união efetiva com a dentina. Para que haja essa união, monômeros resinosos devem penetrar na superfície da dentina previamente condicionada, criando um embricamento micromecânico e a formação da camada híbrida ${ }^{24}$. Assim, o cimento resinoso acaba exercendo a função de dentina artificial, reforçando a estrutura radicular fragilizada pelo tratamento endodôntico. Para da Silva et $\mathrm{al}^{25}$, apesar de efetiva, a resistência de união entre o cimento resinoso e o PFV é um fator crítico para o sucesso de procedimentos endodônticos. Assim, quanto mais adaptado ou justaposto ao canal ficar o pino, melhor.

Por apresentar maiores valores de resistência à tração, o cimento resinoso utilizado no caso possui dupla polimerização (química e física). Segundo Bottino et al. ${ }^{26}$, os PFV permitem a passagem da luz fotopolimerizável até $\mathrm{o}$ ápice, permitindo a polimerização do cimento ao longo de toda extensão desobturada do conduto. Por sua vez, Dallari ${ }^{27}$ (2003) relatou que a quantidade de luz que passa através do pino pode ser incapaz de polimerizar a fina camada de cimento resinoso e a do adesivo aplicado sobre as paredes radiculares. No caso relatado, a fotopolimerização do sistema adesivo foi realizada antes da colocação do cimento resinoso. Grandini et al. $^{28}$ concluíram que desta forma tal procedimento é mais satisfatório quando comparado com a fotopolimerização simultânea envolvendo adesivo e cimento.

Outro aspecto relevante a ser considerado no processo restaurador diz respeito ao cuidado para que a porção coronária do pino não fique exposta ao meio bucal e, assim, possivelmente tenha sua resistência flexural reduzida. Torna-se imprescindível, então, que o material de preenchimento e o remanescente coronário envolvam por completo o pino ${ }^{10}$.

Por meio da técnica utilizada, foi possível obter vantagens quanto à rapidez, simplicidade e previsibilidade, assim como adesão e resistência. Essa relativa facilidade contribuiu para sua escolha no caso apresentado, considerando a preservação da estrutura dentária e a expectativa do paciente, que se mostrava abalado com esse dente fraturado. Por empregar compósitos passíveis de troca ao longo do tempo, ela é indicada justamente para casos como este, em que o jovem paciente ainda se encontra em fase de maturação dos maxilares, não o limitando de realizar tratamentos futuros, a exemplo do ortodôntico.

\section{CONCLUSÃO}

A partir do caso relatado foi possível concluir que, desde que sua indicação e técnica de utilização sejam criteriosas, a restauração direta em resina composta associada à cimentação de PFV no conduto radicular constitui uma rápida e viável alternativa para o restabelecimento estético e funcional de dentes anteriores fragilizados de pacientes hebiátricos, harmonizando o elemento tratado junto aos dentes adjacentes e, consequentemente, melhorando a autoestima do indivíduo.

\section{REFERÊNCIAS}

1. Mazzoleni S, Graf F, Salomon E, Simionato F, Bacci C, Stellini E. Influence of root canal posts on the reattachment of fragments to endodontically treated fractured incisors: an in 
vitro experimental comparison. J Esthet Restor Dent. 2016;28(2):92-101.

2. Andersson L. Epidemiology of traumatic dental injuries. J Endod. 2013;39 (3 Suppl):S2-5.

3. Barreto BCF, Silva GR, Bertaglia PC, Caldeira MMPS, Soares CJ, Martins LRM. Traumatismo dentário na hebiatria: relato de caso clínico. ROBRAC (Online). 2012;21(57):510-14.

4. Flores MT, Andreasen JO, Bakland LK, Feiglin B, Gutmann JL, Oikarinen K et al. Guidelines for the evaluation and management of traumatic dental injuries. Dent Traumatol. 2001;17(2):49-52.

5. Altun C, Tözüm TF, Güven G. Multidisciplinary approach to the rehabilitation of a crown fracture with glass-fibre-reinforced composite: a case report. J Can Dent Assoc. 2008;74(4):363-66.

6. Cvek M, Cleaton-Jones PE, Austin JC, Andreasen JO. Pulp reactions to exposure after experimental crown fractures or grinding in adult monkeys. $\mathbf{J}$ Endod. 1982;8(9):391-97.

7. Lee R, Barrett EJ, Kenny DJ. Clinical outcomes for permanent incisor luxations in a pediatric population. II. Extrusions. Dent Traumatol. 2003;19(5):274-79.

8. Aleisa K, Al-Dwairi Z, Alghabban R, Glickman G, Hsu ML. Effect of cement types and timing of cementation on the retentive bond strength of fiber posts. J Dent Sci. 2012;7(4):367-72.

9. Prado MAA, Kohl JCM, Nogueira RD, GeraldoMartins VR. Retentores intrarradiculares: revisão da literatura. UNOPAR Cient Cienc Biol Saude. 2014;16(1):51-5.

10.Petrie CS, Walker MP. Effect of airborne-particle abrasion and aqueous storage on flexural properties of fiber-reinforced dowels. J Prosthodont. 2012;21(4):296-303.

11. Rocha AC, Cardoso J, Coradini SU. Reforço radicular: relato de caso clínico. Stomatos. 2009;15(28):87-93.

12. Ausiello P, Franciosa P, Martorelli M, Watts DC. Mechanical behavior of post-restored upper canine teeth: a 3D FE analysis. Dent Mater. 2011;27(12):1285-94.

13.Cecchin D, Farina AP, Vitti RP, Moraes RR, Bacchi A, Spazzin AO. Acid etching and surface coating of glass-fiber posts: bond strength and interface analysis. Braz Dent J. 2016;27(2):228-33.

14.Feuser L, Araújo E, Andrada MAC. Pinos de fibra: escolha corretamente. Arq odontol. 2005;41(3):255-62.

15.González-Lluch C, Rodríguez-Cervantes PJ, Sancho-Bru JL, Pérez-González A, BarjauEscribano A, Vergara-Monedero $M$ et al. Influence of material and diameter of prefabricated posts on maxillary central incisors restored with crown. J Oral Rehabil. 2009;36(10):737-47.
16. Gomes GM, Gomes OM, Reis A, Gomes JC, Loguercio AD, Calixto AL. Effect of operator experience on the outcome of fiber post cementation with different resin cements. Oper Dent. 2013;38(5):555-64.

17. Sorrentino R, Salameh Z, Zarone F, Tay FR, Ferrari M. Effect of post-retained composite restoration of MOD preparations on the fracture resistance of endodontically treated teeth. J Adhes Dent. 2007;9(1):49-56.

18.Rosentritt M, Fürer C, Behr M, Lang R, Handel G. Comparison of in vitro fracture strength of metallic and tooth-coloured posts and cores. J Oral Rehabil. 2000;27(7):595-601.

19. Schwartz RS, Fransman R. Adhesive dentistry and endodontics: materials, clinical strategies and procedures for restoration of access cavities: a review. J Endod. 2005;31(3):151-65.

20.Egilmez F, Ergun G, Cekic-Nagas I, Vallittu PK, Lassila LV. Influence of cement thickness on the bond strength of tooth-colored posts to root dentin after thermal cycling. Acta Odontol Scand. 2013;71(1):175-82.

21.Baba NZ, Golden G, Goodacre CJ. Nonmetallic prefabricated dowels: a review of compositions, properties, laboratory, and clinical test results. J Prosthodont. 2009;18(6):527-36.

22.Clavijo VGR, Souza NC, Andrade MF, Susin AH. Pinos anatômicos: uma nova perspectiva clínica. Rev dental press estét. 2006;3(3):100-21.

23.Conceição EN. Dentística: saúde e estética. Porto Alegre: Artmed; 2007.

24.Maurício P, Reis J. Tendências na reabilitação de dentes com tratamento endodôntico em prótese fixa. Revista OMD. 2014;2-8.

25.Bastos PCA, Faria DE, Bridi EC, Amaral FLB, França FMG, Flório FM et al. Push-out bond strength and sealing ability of etch-and-rinse and self-etching adhesives used for fiberglass dowel bonding at different depths of the root canals. Rev odontol UNESP. 2011;40(4):174-81.

26.da Silva NR, Aguiar GCR, Rodrigues MP, Bicalho AA, Soares PBF, Veríssimo $\mathrm{C}$ et al. Effect of resin cement porosity on retention of glass-fiber posts to root dentin: an experimental and finite element analysis. Braz Dent $\mathrm{J}$. 2015;26(6):630-36.

27.Bottino MA, Quintas AF, Miyashita E, Giannini V. Núcleos. In: Estética em reabilitação oral: metal free. São Paulo: Artes Médicas; 2011.

28.Dallari, A. Comunicação pessoal, Brescia, 2001. In: Scotti, R. Pinos de fibra-considerações teóricas e aplicações clinicas. Artes Médicas; 2003.

29. Grandini S, Sapio S, Goracci C, Monticelli F, Ferrari M. A onestep procedure for luting glass fibre posts: an SEM evaluation. Int Endod J. 2004;37(10):679-86. 


\section{CONFLITO DE INTERESSES}

Os autores declaram não haver conflitos de interesse.

\section{AUTOR PARA CORRESPONDENCIA}

\section{Maria Helena Chaves de Vasconcelos Catão}

mhelenact@zipmail.com.br

Submetido em 27/07/2018

Aceito em 04/10/2018 